\title{
Methods of Design-Oriented Analysis: The Quadratic Equation Revisited
}

\author{
R.D. Middlebrook \\ California institute of Technology
}

The conventional formula for quadratic roots suffers from two defects: it is High Entropy, and it is computationally inaccurate when two real roots are widely separated. An improved formula is suggested that overcomes both defects. Both roots are expressed in terms of a single parameter $\mathrm{F}$ that contains the radical sign and is a unique function of the single parameter $\mathrm{Q}$ that determines the nature of the roots.Both roots are computed in terms of $\mathrm{F}$ with the same computational accuracy, and the Low Entropy format exposes the useful design-oriented result that, for well-separated real roots, $\mathrm{F}$ approaches unity so that the radical disappears and both roots reduce to simple ratios of the original quadratic coefficients.

\section{Introduction}

It might be thought that the quadratic equation would be beneath the notice of this conference. However, when reminded of the conventional formulation in terms of the three $a, b, c$ coefficients, even graduate students often give depressing answers to the following questions:

\#1. How many independent parameters determine the roots?

\#2. How many independent parameters determine whether the roots are real or complex?

\#3. Did you know that real roots, to a good approximation, can be expressed as simple ratios of the $a, b, c$ coefficients?

It is suggested here that enhanced understanding, and hence more efficient application, of the quadratic equation may be imparted in the context of Design-Oriented Analysis, proposed at the FIE'91 conference [1].

Design-Oriented Analysis is a paradigm for teaching design as an integral part of analysis. This is in contrast to the more usual approach in which "design" is an appendage to detailed and lengthy treatment of "analysis." To review the theme of [1]:

Design is the reverse of analysis: one starts with the Answer (the Specification), and one has to work back to the beginning (circuit configuration and element values). Therefore, only analysis that can be worked backwards is worth doing. This is Design-Oriented Analysis.

Design-Oriented Analysis is the process of guiding and controlling the algebra so that the result is a Low Entropy Expression, defined as one in which the terms are ordered, or grouped, so that additional insight is obtained into the relative importance of the various contributions to the result.

Engineers always have to solve for unknowns determined by an insufficient number of equations. A Low Entropy Expression allows more than one piece of information to be extracted from one equation, and helps to substitute for the missing equations that would be needed to solve formally for the number of unknowns.

\section{Defects of the Conventional Quadratic Formula}

The standard-notation quadratic is

$$
\mathrm{y}=a \mathrm{x}^{2}+b \mathrm{x}+c=a\left(\mathrm{x}-\mathrm{x}_{1}\right)\left(\mathrm{x}-\mathrm{x}_{2}\right)
$$

whose conventional root formula is

$$
\mathrm{x}_{1}, \mathrm{x}_{2}=\frac{-b \pm \sqrt{b^{2}-4 a c}}{2 a}
$$

This familiar formula, which we all know and love, suffers from two disadvantages:

Defect \#1. It is a High Entropy Expression, in that little or no insight is obtained into how the values of $a, b, c$ influence the result.

Defect $\hbar 2$. It is numerically inaccurate, in that for real roots the smaller root is computed with lower accuracy than the larger root; the accuracy declines the smaller that root is relative to the other. The origin of this effect is use of an algorithm that requires computation of the small difference of large numbers, a concept that students hear about in more advanced courses, but often fail to recognize in the lowly quadratic.

Let us examine these defects, and attempt to overcome them.

A small rearrangement effects significant improvement already with respect to Defect \#1:

$$
\mathrm{x}_{1}, \mathrm{x}_{2}=-\frac{b}{2 a} \pm \sqrt{\left(\frac{b}{2 a}\right)^{2}-\left(\frac{c}{a}\right)}
$$

In this Lower-Entropy format it is immediately seen that the two roots are determined by only two independent parameters, (b/a) and (c/a); the third parameter, $a$, is merely a normalizing factor on $\mathrm{y}$, and need not be considered further. Another small rearrangement leads to a Low Entropy form especially suited for complex roots:

$$
\mathrm{x}_{1}, \mathrm{x}_{2}=-\frac{b}{2 a} \pm j \sqrt{\left(\frac{c}{a}\right)-\left(\frac{b}{2 a}\right)^{2}}
$$

Before discussion of a different Low Entropy form more suitable for real roots, let us consider Defect \#2.

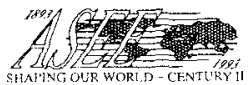

1992 Frontiers in Education Conference

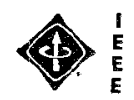


Computational inaccuracy creeps in when $(c / a)$ is much smaller than $(b / 2 a)^{2}$, in the form of the old nemesis Small Difference of Large Numbers:

$$
\begin{aligned}
& \mathrm{x}_{1} \equiv-\frac{b}{2 a}\left[1-\sqrt{1-\frac{(c / a)}{(b / 2 a)^{2}}}\right] \\
& \mathrm{x}_{2} \equiv-\frac{b}{2 a}\left[1+\sqrt{1-\frac{(c / a)}{(b / 2 a)^{2}}}\right]
\end{aligned}
$$

In this situation, $x_{1}$ is computed in terms of a factor (1/2) minus something almost $(1 / 2)$, thereby multiplying the calculator roundoff error. This Achilles heel is not possessed by the root $\mathrm{x}_{2}$. Thus, real roots are computed with unequal accuracy.

Students tend to think that because engineers may be satisfied with answers that are accurate to $10 \%$, or at best $1 \%$, and that they have at least 10 -digit pocket calculators, they don't need to worry about computational accuracy, certainly not with respect to the quadratic formula. A simple numerical example destroys this faith.

Consider a quadratic in $\mathrm{z}$ with $a=c=1, b=45,000$

$$
z^{2}+b z+1=0
$$

The root $z_{1}$ from Eq. (2), call it $z_{1 a}$, is computed on an HP15C as

$$
\begin{aligned}
z_{\text {la }} & =\frac{-b+\sqrt{b^{2}-4}}{2} \\
& =2.000,000,000 \times 10^{-5}
\end{aligned}
$$

[The HP15C is a 10-digit calculator, and it takes a little effort to get it to display all 10 digits, since the last two are normally preempted by the exponent.] However, the same root $z_{1}$ from Eq. (5), call it $z_{i b}$, is computed as

$$
\begin{aligned}
z_{1 b} & =-\frac{b}{2}\left[1-\sqrt{1-4 / b^{2}}\right] \\
& =2.250,000,000 \times 10^{-5}
\end{aligned}
$$

This is a bit disturbing (one hopes): The two results for the same root, both computed from exact formulas on the same calculator, differ by more than $10 \%$. This leads to an even more disturbing question: Which result is "right"? Worse, perhaps they're both wrong.

The difference between the two results must arise from the computational algorithm, which in this case refers to the format of the original expression. Let's work on the analytic format and see if we can persuade it to reveal the "right" answer.

$$
\text { Rearrange Eq. (6) to }
$$

$$
z=-\frac{1}{b}\left(1+z^{2}\right)
$$

The root $z_{1}$ under examination is known from Eq. (7) or (8) to be much less than 1 , regardless of whether neither result is correct. Therefore, a zeroth-order result, call it $\mathrm{z}_{\mathrm{lc}}$, is obtained by dropping the $z^{2}$ on the right-hand side of Eq. (9):

$$
\mathrm{z}_{\mathrm{lc}}=\frac{1}{b}=-2.222,222,222 \times 10^{-5}
$$

A first-order result, call it $z_{1 \mathrm{~d}}$, is obtained by substitution of the zeroth-order $z_{10}$ into the right-hand side of Eq. (9):

$$
\begin{aligned}
z_{1 \mathrm{~d}}= & -\frac{1}{b}-\frac{1}{b^{3}} \\
= & -2.222,222,222 \quad \times 10^{-5} \\
& -0.000,000,001,097,393,690 \times 10^{-5}(11)
\end{aligned}
$$

Since the last (10th) digit of each of the two numbers to be summed is subject to calculator roundoff error, the following statement can be made: The "right" answer is

$$
\begin{gathered}
z_{1}=-2.222,222,22 \times 10^{-5}, \text { correct to } 9 \\
\text { significant figures }
\end{gathered}
$$

Students sometimes take a little time to decide whether they really believe this statement. When they are ready to continue, it can be pointed out that both previous results $z_{1 a}$ and $\mathrm{z}_{1 \mathrm{~b}}$ are wrong, $\mathrm{z}_{1 \mathrm{a}}$ by $10 \%$, and $\mathrm{z}_{\mathrm{lb}}$ by more than $1 \%$.

Students may be curious enough to repeat the three calculations for $\mathrm{z}_{1 \mathrm{a}}, \mathrm{z}_{1 \mathrm{~b}}$, and the "right" $\mathrm{z}_{1}$ for other values of $b$, say 40,000 plus 1,000 increments to 50,000 . The errors in $z_{1 a}$ and $z_{1 \mathrm{~b}}$ are found to be scattered; sometimes $z_{1 \mathrm{a}}$ has greater error, sometimes $z_{1 b}$. An 8-digit calculator gives zero for $z_{1 a}$ and $z_{1 b}$; rather a large percentage error!

Clearly, the computational algorithm makes a significant difference in the accuracy of the result. The punch-line, however, is still to come: Both exact formulas, Eqs. (7) and (8), gave wrong answers, and the right answer was obtained from an approximate formula, Eq. (11)!

With the accuracy defect thus exposed, students are more receptive to the proposal that a more useful, LowerEntropy, format for the quadratic roots might be available.

\section{Improved, Low Entropy, Quadratic Formula}

The key to an improved result is to construct an algorithm in which the subtraction of two almost-equal quantities is performed analytically, before introduction of numerical values with consequent computational roundoff error. Let us go back to the original format, Eq. (2), and write the two roots as

$$
\begin{aligned}
& \mathrm{x}_{1}=-\frac{b}{a}\left[\frac{1}{2}-\frac{1}{2} \sqrt{1-\frac{4 a c}{b^{2}}}\right] \\
& \mathrm{x}_{2}=-\frac{b}{a}\left[\frac{1}{2}+\frac{1}{2} \sqrt{1-\frac{4 a c}{b^{2}}}\right]
\end{aligned}
$$

The problem resides in the computation of $\mathrm{x}_{1}$, so let us exhume another algebraic procedure from high school, "rationalizing the denominator," but this time we'll rationalize the numerator of $x_{1}$ :

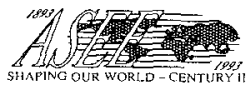

1992 Frontiers in Education Conference

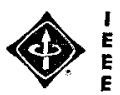




$$
\begin{aligned}
\mathrm{x}_{\mathrm{1}} & =-\frac{b}{a} \frac{\left[\frac{1}{2}-\frac{1}{2} \sqrt{1-\frac{4 a c}{b^{2}}}\right]\left[\frac{1}{2}+\frac{1}{2} \sqrt{1-\frac{4 a c}{b^{2}}}\right]}{\left[\frac{1}{2}+\frac{1}{2} \sqrt{1-\frac{4 a c}{b^{2}}}\right]} \\
& =-\frac{b}{a} \frac{\frac{1}{4}-\frac{1}{4}\left(1-\frac{4 a c}{b^{2}}\right)}{\left[\frac{1}{2}+\frac{1}{2} \sqrt{1 \frac{4 a c}{b^{2}}}\right]}
\end{aligned}
$$

The crucial step follows: $(1 / 4)-(1 / 4)=0$ exactly, and the "small difference" $a c / b^{2}$ is left exposed analytically, before substitution of numbers and consequent roundoff error:

$$
\begin{aligned}
& \mathrm{x}_{1}=-\frac{c}{b} \frac{1}{\left[\frac{1}{2}+\frac{1}{2} \sqrt{1-\frac{4 a c}{b^{2}}}\right]} \\
& \mathrm{x}_{2}=-\frac{b}{a}\left[\frac{1}{2}+\frac{1}{2} \sqrt{1 \frac{4 a c}{b^{2}}}\right]
\end{aligned}
$$

where $x_{2}$ from (13) has been repeated in order to display the two roots together. In other words, this format for $x_{1}$ is superior to that of Eq. (13) because Defect \#2, the Small Difference of Large Numbers, is avoided.

This derivation is longer than necessary in order that the crucial step could be exposed pedagogically. A shorter derivation can be used to introduce conveniently some new notation that ultimately assists in lowering the entropy, thus also avoiding Defect \#1.

Let us go back this time to one step beyond the original format, Eq. (13), and introduce two quantities $Q$ and $F$ such that

$$
\mathrm{x}_{2}=-\frac{b}{a} \mathrm{~F}
$$

where

$$
\mathrm{F}=\frac{1}{2}+\frac{1}{2} \sqrt{1-4 \mathrm{Q}^{2}}
$$

in which

$$
\mathrm{Q} \equiv \frac{\sqrt{a c}}{b} \quad\left(=\frac{\sqrt{c / a}}{b / a}\right)
$$

The root $\mathrm{x}_{2}$ given by (16) suffers from no problem; it is $\mathrm{x}_{1}$ that needs work. Instead of manipulating Eq. (13) in $x_{1}$, let us dust off another property of the quadratic known (at least fleetingly) to high school students: the product of the two roots equals the constant term of the quadratic, or

$$
a \mathrm{x}_{1} \mathrm{x}_{2}=c
$$

as easily seen from Eq. (1). Hence, since $x_{2}$ is already known in a good shape (the Low Entropy form of Eq. (16)), $x_{1}$ is immediately given as

$$
\mathrm{x}_{1}=\frac{c}{a} \frac{1}{\mathrm{x}_{2}}=-\frac{c}{a} \frac{a}{b} \frac{1}{\mathrm{~F}}
$$

or

$$
\mathrm{x}_{1}=-\frac{c}{b} \frac{1}{\mathrm{~F}} \quad \mathrm{x}_{2}=-\frac{b}{a} \mathrm{~F}
$$

where again the results for both roots have been displayed together.

The two results of Eqs. (20) are the same as those of Eqs. (15), but with the added notational economy of $F$ and $Q$ defined by Eqs. (17) and (18), in which the computational accuracy Defect \#2 has been eliminated.

The additional benefits leading to elimination of Defect \#1 can now be examined, since they are embodied in the physical interpretation of $\mathrm{F}$ and $\mathrm{Q}$.

$$
\begin{aligned}
& Q>0.5: \text { complex pair } \\
& Q=0.5: 2 \text { equal real roots } \\
& Q<0.5: 2 \text { unequal real roots }
\end{aligned}
$$

It is no accident that the symbol $Q$ was chosen because it is the same as the Q-factor of an LC resonant tank. Students who are uncertain about the answer to the foregoing question \#2 immediately realize that they actually know the answer, when the question is put in the context of resonance.

The biggest payoff from the Low Entropy format of (20) arises from the function F: the smaller $Q$ becomes, the closer $F$ approaches the limiting value unity, or

$$
\mathrm{Q}<<0.5: \quad \mathrm{F} \rightarrow 1
$$

There is an immediate consequence: in this limit, each of the two quadratic roots becomes a simple ratio of the original $a, b, c$ coefficients, or

$$
\text { and } \left.\quad \begin{array}{l}
\mathrm{x}_{1} \approx-\frac{c}{b} \\
\mathrm{x}_{2} \approx-\frac{b}{a} \\
\frac{\mathrm{x}_{1}}{\mathrm{x}_{2}} \approx \mathrm{Q}^{2}
\end{array}\right] \quad \begin{aligned}
& \mathrm{Q}<0.5 \\
& (\mathrm{~F} \rightarrow 1)
\end{aligned}
$$

Question \#3 of the Introduction is thereby answered: as long as the ratio of the two real roots is sufficiently small (they are sufficiently well-separated), each root is a simple ratio of the original quadratic coefficients, and no radical sign is needed in the analytic expression.

It is now clear why the factor $1 / 2$ was included in the definition of $F$, instead of leaving it outside: a limiting value for $F$ of 1 is simpler and more easily remembered and used than some nonunity value (it is a Low Entropy Expression).

It remains to be seen how rapidly $F$ approaches 1 as $Q$ decreases. As seen from the graph of $F$ vs $Q$ in Fig. 1, the approach is very rapid. Real roots occur for $\mathrm{Q} \leq 0.5$, and for $\mathrm{Q}=0.3, \mathrm{~F}=1$ with $<10 \%$ error. All this information in Fig. 1 is

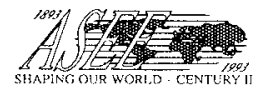

1992 Frontiers in Education Conference 


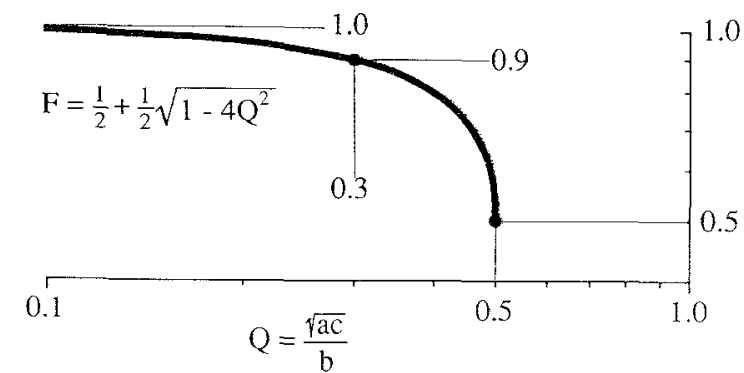

Fig.1. The function $\mathrm{F}$ rapidly approaches unity as $\mathrm{Q}$ drops below 0.5 , making the radical sign disappear for well-separated real roots.

easily memorized and allows the following qualitative statement to be made: except for a narrow range of $Q$ just below 0.5 , whenever the quadratic roots are real they may be approximated by Eqs. (21). The approximation improves the smaller the value of $\mathrm{Q}$, that is, the more unequal are the roots.

$$
A=\frac{R_{L}+C_{1} R_{2} R_{L} s}{\left[R_{1}+R_{L}\right]+\left[C_{1}\left(R_{1} R_{2}+R_{L} R_{2}+R_{1} R_{L}\right)+C_{2} R_{1} R_{L}\right] s+\left[C_{1} C_{2} R_{1} R_{2} R_{L}\right] s^{2}}
$$

[This contrasts with Defect $\# 2$ of the conventional format, in which the accuracy worsens the smaller the value of Q.]

A summary of the recommended formats is shown in Table 1. The results are Low Entropy in that the radical is inside the function $F$, whose value for real roots is confined to the narrow range 0.5 to 1 , and the same $F$ appears as a factor in both roots, which otherwise are simple ratios of the quadratic coefficients. Thus, computational roundoff error remains in the last digit, and both roots are computed to the same percentage accuracy. The exact results are of course valid for any value of $\mathrm{Q}$, and a complex $\mathrm{F}$ implies a complex root pair.

The Low Entropy nature of the results is especially beneficial when the roots are real and sufficiently well-separated that $\mathrm{F}$ can be taken equal to 1 . Then, no radical appears in the expression for either root, making analytic factorization of the quadratic useful. Moreover, the degree of approximation is easily tracked by evaluating the actual value of $F$, and affects each root proportionally.

Taking $F \approx 1$ for real roots could be considered a zeroth order approximation for $\mathrm{F}$. If desired, a first-order

$$
A=\frac{R_{L}}{R_{1}+R_{L}} \frac{1+C_{1} R_{2} s}{1+\left[C_{1}\left(R_{2}+R_{\|} \| R_{L}\right)+C_{2}\left(R_{1} \| R_{L}\right)\right] s+\left[C_{1} C_{2} R_{2}\left(R_{1} \| R_{L}\right)\right] s^{2}}
$$

approximation can be found by binomial expansion of the radical in (17), which gives $F \approx 1-Q^{2}$, and which also could be sketched in Fig. 1. At $Q=0.3,1-Q^{2}=0.91$, only about a $1 \%$ error from the actual value $\mathrm{F}=0.90$.

It should be pointed out that the function $\mathrm{F}$ of Eq. (17) has been plotted in Fig. 1 on the assumption that all three $a, b, c$ coefficients of the original quadratic are positive. If $a$ or $c$ is negative, $Q^{2}$ is negative; then, $F$ exceeds unity and the roots are always real regardless of the value of $Q^{2}$. The shape of $F$ is different from that shown in Fig. 1, but all the expressions of (17), (18), and (20) remain correct.

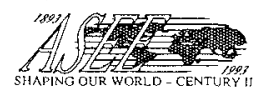

1992 Frontiers in Education Conference coefficient.

which exhibits several Low Entropy features. First, the two polynomials each has a leading term of 1 , exposing the initial coefficient $R_{L} /\left(R_{1}+R_{L}\right)$ as the zero-frequency gain $A_{0}$, obvious from the model of Fig. 3. Second, the resistances otherwise appear as series/parallel combinations, exposing their relative importances. Third, the coefficient of the denominator s term is seen to be the sum of two contributions, and it is obvious by inspection, without even substituting numbers, that the $C_{2}$ contribution is negligible compared to that of the $\mathrm{C}_{1}$ term, and therefore may be dropped with less than $2 \%$ error in the

This is a High Entropy Expression with respect to the concepts elements, and conveys no urious obtained by direct insertion of numerical values.

entropy, a better method, again following the recommendation , is to do some of the algebra on the circuit diagram before

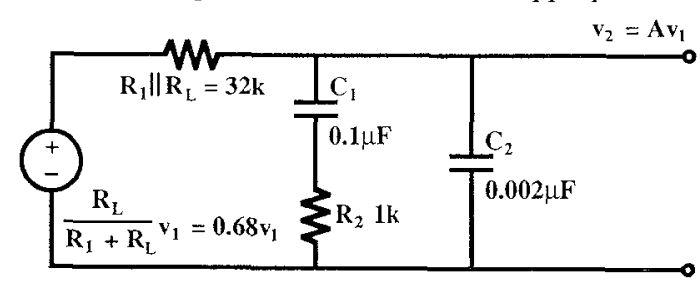

Fig.3. A Lower Entropy version of the circuit example of Fig.2. to eliminate one of the loops by Thevenin reduction, namely, to convert $\mathrm{v}_{1}, \mathrm{R}_{1}$, and $\mathrm{R}_{\mathrm{L}}$ into a Thevenin equivalent, as shown in Fig. 3. The result now is

example to illustrate the improved formula for

Standard analysis leads to the usual result as a ratio of polynomials in complex frequency s: 
This is an example of another Method of DesignOriented Analysis: Use of Numerical Values to Justify Analytic Approximations. The principle is that non-dropped terms are retained in analytic form, so that a simpler but still general result is maintained. The advantage is that numerical values can be changed later in order to meet some design specification, and it is merely necessary to go back and check that the approximation is still valid.

In the present example, the reduced expression for the network gain is

$$
A=\frac{R_{L}}{R_{1}+R_{L}} \frac{1+C_{1} R_{2} s}{1+\left[C_{1}\left(R_{2}+R \| R_{L}\right)\right] s+\left[C_{1} C_{2} R_{2}\left(R \| R_{L}\right)\right] s^{2}}
$$

This function contains a denominator quadratic in $\mathrm{s}$, whose roots $\omega_{1}$ and $\omega_{2}$ are the two poles of the function.

Substitution into the conventional formula (2) gives

$$
\omega_{1}, \omega_{2}=\frac{2}{C_{1}\left(R_{2}+R_{\|} \| R_{L}\right) \pm \sqrt{C_{1}^{2}\left(R_{2}+R_{\|} \| R_{L}\right)^{2}-4 C_{1} C_{2} R_{2}\left(R_{1} \| R_{L}\right)}}
$$

This is a High Entropy Expression, and gives no insight into the influence of any element on the result. It isn't even immediately obvious whether the roots are real or complex. Indeed, it was a waste of time even writing this expression, because all it's good for is for substitution of numerical values, which might just as well have been done in the original quadratic. Furthermore, such a procedure will result in the two roots being computed with different (and unknown) degrees of accuracy.

Instead, let us use the Low Entropy Expressions from Table 1, which leads to

$$
\begin{aligned}
& \omega_{1}=\frac{1}{C_{1}\left(R_{2}+R_{1} \| R_{L}\right)} \frac{1}{F} \\
& \omega_{2}=\frac{C_{1}\left(R_{2}+R_{1} \| R_{L}\right)}{C_{1} C_{2} R_{2}\left(R_{1} \| R_{L}\right)} F=\frac{1}{C_{2}\left(R_{2}\left\|R_{1}\right\| R_{L}\right)} F
\end{aligned}
$$

Note that the Low Entropy format already gives a vastly superior analytic result even before $F$ is known, since $F$ is a mere number (between 0.5 and 1 ).

Evaluation of Q gives

$$
\mathrm{Q}=\frac{\sqrt{\mathrm{C}_{1} \mathrm{C}_{2} \mathrm{R}_{2}\left(\mathrm{R}_{1} \| \mathrm{R}_{\mathrm{L}}\right)}}{\mathrm{C}_{1}\left(\mathrm{R}_{2}+\mathrm{R}_{1} \| \mathrm{R}_{\mathrm{L}}\right)}=\sqrt{\frac{\mathrm{C}_{2}}{\mathrm{C}_{1}} \frac{\mathrm{R}_{4}\left\|\mathrm{R}_{\|}\right\| \mathrm{R}_{\mathrm{L}}}{\mathrm{R}_{2}+\mathrm{R}_{\Downarrow} \| \mathrm{R}_{\mathrm{L}}}}
$$

It is obvious by inspection, without actual substitution of numbers, that $\mathrm{Q}$ is going to be much less than 0.5 , and so the real-root approximation $F=1$ can safely be adopted. Nevertheless, to press on with the computation,

$$
\mathrm{Q}=0.024
$$

which meets the $\mathrm{Q} \ll 0.5$ inequality by more than a factor of 20 , thus amply justifying the real-root approximation $F=1$.
Actually,

$$
\begin{aligned}
F & =\frac{1}{2}+\frac{1}{2} \sqrt{1-4(0.024)^{2}} \\
& =0.9994
\end{aligned}
$$

and so $F$ can be taken equal to 1 with less than $0.1 \%$ error. With the same percentage error in each root, the two roots of the quadratic, the poles of the network gain, are

$$
\begin{aligned}
& \omega_{1}=\frac{1}{C_{1}\left(R_{2}+R_{I} \| R_{L}\right)} \\
& \omega_{2}=\frac{1}{C_{2}\left(R_{2}\left\|R_{\Downarrow}\right\| R_{L}\right)}
\end{aligned}
$$

so that the complete gain expression A can be written in factorec pole/zero form:

$$
A=A_{0} \frac{\left(1+\frac{s}{\omega_{2}}\right)}{\left(1+\frac{s}{\omega_{1}}\right)\left(1+\frac{s}{\omega_{2}}\right)}
$$

A comparison between the above Low Entropy Expressions of (30) for $\omega_{1}$ and $\omega_{2}$ and the High Entropy Expressions of (25) is no contest. One conclusion leaps to the eye: $\mathrm{C}_{1}$ determines $\omega_{1}$ and $\mathrm{C}_{2}$ determines $\omega_{2}$, and there is no interaction. One would never know this by gazing at $\mathrm{Eq}$. (25): it looks as though $\mathrm{C}_{1}$ and $\mathrm{C}_{2}$ are inextricably intertwined, both determining both poles.

From an analysis point of view, the Low Entropy format obviously outdistances the conventional High Entropy format. The bottom line, of course, is the design point of view, from which it is equally obvious that the Low Entropy format wins hands down. In a design context, $\omega_{1}$ and $\omega_{2}$ would be given (or derived from) the specifications, and the problem would be to select element values, primarily $C_{1}$ and $C_{2}$. This is simply achieved by separate inversion of Eqs. (30), but would be incomparably more difficult from the High Entropy Eqs. (25). The same is true to a lesser extent if there is another constraint on $C_{1}$ and $C_{2}$, and the specified roots must be achieved by selection of resistor values: the Low Entropy Eqs. (30) reveal that $\omega_{1}$ is dominated by $R_{1} \| R_{L}, \omega_{2}$ by $R_{2}$. Again, Eqs. (25) would be no help.

\section{Alternative Formats of the Low Entropy Quadratic Formula}

As usual, there are many possible Low Entropy formats for a given expression; the choice depends on what features of the result are to be exposed. The above discussion adopted the conventional "high school" format of Eq. (1) as a starting point, in which the $a$ coefficient was extracted as a normalizing factor for $y$.

It is often convenient to adopt the constant term $c$ as a normalizing parameter so that, with reversed sequence of terms, the original quadratic becomes

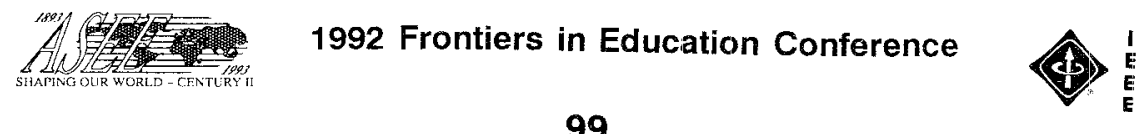




$$
\mathrm{y}=c+b \mathrm{x}+a \mathrm{x}^{2}=c\left(1-\frac{\mathrm{x}}{\mathrm{x}_{1}}\right)\left(1-\frac{\mathrm{x}}{\mathrm{x}_{2}}\right)
$$

A case in point would be the previous network example with a quadratic in the denominator of the transfer function, in which $\mathrm{x}$ is complex frequency $s$, and $-x_{1}$ and $-x_{2}$ are the poles $\omega_{1}$ and $\omega_{2}$

Since $c$ is now a normalizing parameter for $y$, and since also the remaining two independent parameters that determine the two roots are $b / c$ and $a / c$, one might introduce two new symbols $a_{1} \equiv b / c$ and $a_{2} \equiv a / c$ so that the original quadratic can be rewritten as

$$
\mathrm{y} / c=1+a_{1} \mathrm{x}+a_{2} \mathrm{x}^{2}=\left(1-\frac{\mathrm{x}}{\mathrm{x}_{1}}\right)\left(1-\frac{\mathrm{x}}{\mathrm{x}_{2}}\right)
$$

The previous discussion leading to the Low Entropy results can now be repeated in terms of the alternative parameters $a_{1}$ and $a_{2}$. The results are displayed in Table 2, and correspond directly to those in Table 1 .

Two points are worthy of note. First, the function $F$ in terms of $Q$ remains the same, shown in Fig. 1. Second, the real-root approximate factorization

$$
\frac{\mathrm{y}}{c}=\left(1+a_{1} \mathrm{x}\right)\left(1+\frac{a_{2}}{a_{1}} \mathrm{x}\right)
$$

is particularly simple and easy to remember: the first factor is identical to the first two terms of the original quadratic; the coefficient of $x$ in the second term is the ratio of the third to the second coefficient in the original quadratic.

Another useful Low Entropy manipulation of the format is to normalize both $\mathrm{x}$ and $\mathrm{y}$ :

$$
\begin{aligned}
\frac{y}{c} & =1+\frac{b}{c} \mathrm{x}+\frac{a}{c} \mathrm{x}^{2} \\
& =1+\frac{b}{c} \sqrt{\frac{c}{a}}\left(\sqrt{\frac{a}{c}} \mathrm{x}\right)+\left(\sqrt{\frac{a}{c}} \mathrm{x}\right)^{2} \\
& =1+\frac{b}{\sqrt{a c}}\left(\sqrt{\frac{a}{c}} \mathrm{x}\right)+\left(\sqrt{\frac{a}{c}} \mathrm{x}\right)^{2}
\end{aligned}
$$

This form is particularly suitable when $\mathrm{x}$ is complex frequency $\mathrm{s}$, and $\sqrt{c / a}$ is some normalizing frequency $\omega_{0}$. Since the coefficient of the middle term is recognized as $1 / \mathrm{Q}$, by Eq.(18), the resulting format is

$$
f\left(\frac{\mathrm{s}}{\omega_{0}}\right)=1+\frac{1}{\mathrm{Q}}\left(\frac{\mathrm{s}}{\omega_{0}}\right)+\left(\frac{\mathrm{s}}{\omega_{0}}\right)^{2}
$$

The single parameter $Q$ that determines the nature of the roots is now featured at the outset, and is the only parameter needed to express the relation between the normalized variables. Table 3 summarizes the Low Entropy results in terms of the single parameter Q.

An example is a low-pass LC filter, which has a voltage transfer function $\mathrm{A}$ of the standard second-order form

\begin{tabular}{|c|c|c|}
\hline Quadratic: & \multicolumn{2}{|c|}{$f\left(\frac{\mathrm{s}}{\omega_{0}}\right)=1+\frac{1}{\mathrm{Q}}\left(\frac{\mathrm{s}}{\omega_{0}}\right)+\left(\frac{\mathrm{s}}{\omega_{0}}\right)^{2}=\left(1+\frac{\mathrm{s}}{\omega_{1}}\right)\left(1+\frac{\mathrm{s}}{\omega_{2}}\right)$} \\
\hline Definitions: & \multicolumn{2}{|c|}{$F=\frac{1}{2}+\frac{1}{2} \sqrt{1-4 Q^{2}}$} \\
\hline \multirow[b]{2}{*}{ Roots: } & Exact & $\begin{array}{l}\text { Well-separated real roots: } \\
\qquad \mathrm{Q} \ll 0.5, \mathrm{~F} \approx 1\end{array}$ \\
\hline & $\begin{array}{c}\omega_{1}=\mathrm{Q} \omega_{0} \frac{1}{\mathrm{~F}}, \quad \omega_{2}=\frac{\omega_{0}}{\mathrm{Q}} \mathrm{F} \\
\frac{\omega_{1}}{\omega_{2}}=\frac{\mathrm{Q}^{2}}{\mathrm{~F}^{2}}\end{array}$ & $\begin{array}{c}\omega_{1}=\mathrm{Q} \omega_{0}, \quad \omega_{2}=\frac{\omega_{0}}{\mathrm{Q}} \\
\frac{\omega_{1}}{\omega_{2}}=\mathrm{Q}^{2}\end{array}$ \\
\hline Factorization: & $f\left(\frac{s}{\omega_{0}}\right)=\left(1+\frac{s}{\left.Q \omega_{\%}\right)}\right)\left(1+\frac{s}{F \omega_{\%}}\right)$ & $f\left(\frac{s}{\omega_{0}}\right)=\left(1+\frac{s}{Q \omega_{0}}\right)\left(1+\frac{s}{\omega_{0} / Q}\right)$ \\
\hline
\end{tabular}

\begin{tabular}{|l|c|c|}
\hline Quadratic: & \multicolumn{1}{|c|}{$\mathrm{y}=a \mathrm{x}^{2}+b \mathrm{x}+c=a\left(\mathrm{x}-\mathrm{x}_{1}\right)\left(\mathrm{x}-\mathrm{x}_{2}\right)$} \\
\hline Definitions: & $\mathrm{Q}=\frac{\sqrt{a c}}{b}, \quad \mathrm{~F}=\frac{1}{2}+\frac{1}{2} \sqrt{1-4 \mathrm{Q}^{2}} \quad$ (Fig. 1) \\
\hline \multirow{2}{*}{ Roots: } & $\begin{array}{c}\text { Well-separated real roots: } \\
\mathrm{Q}<0.5, \mathrm{~F} \approx 1\end{array}$ \\
\cline { 2 - 3 } & $\mathrm{x}_{1}=-\frac{c}{b} \frac{1}{\mathrm{~F}}, \quad \mathrm{x}_{2}=-\frac{b}{a} \mathrm{~F}$ & $\mathrm{x}_{1}=-\frac{c}{b}, \quad \mathrm{x}_{2}=-\frac{b}{a}$ \\
$\frac{\mathrm{x}_{1}}{\mathrm{x}_{2}}=\frac{\mathrm{Q}^{2}}{\mathrm{~F}^{2}}$ & $\frac{\mathrm{x}_{1}}{\mathrm{x}_{2}}=\mathrm{Q}^{2}$ \\
\hline Factorization: & $\mathrm{y}=a\left(\mathrm{x}+\frac{c}{b} \frac{1}{\mathrm{~F}}\right)\left(\mathrm{x}+\frac{b}{a} \mathrm{~F}\right)$ & $\mathrm{y}=a\left(\mathrm{x}+\frac{c}{b}\right)\left(\mathrm{x}+\frac{b}{a}\right)$ \\
\hline
\end{tabular}

Table 1. Improved quadratic formulas in terms of the original three a.b.c coefficients.

\begin{tabular}{|l|c|c|}
\hline Quadratic: & \multicolumn{2}{|c|}{$\frac{\mathrm{y}}{c}=1+a_{1} \mathrm{x}+a_{2} \mathrm{x}^{2}=\left(1-\frac{\mathrm{x}}{\mathrm{x}_{1}}\right)\left(1-\frac{\mathrm{x}}{\mathrm{x}_{2}}\right)$} \\
\hline Definitions: & $\mathrm{Q} \equiv \frac{\sqrt{a_{2}}}{a_{1}}, \quad \mathrm{~F}=\frac{1}{2}+\frac{1}{2} \sqrt{1-4 \mathrm{Q}^{2}} \quad$ (Fig. 1) \\
\hline \multirow{2}{*}{ Roots: } & $\begin{array}{c}\text { Well-separated real roots: } \\
\mathrm{Q} \ll 0.5, \mathrm{~F} \approx 1\end{array}$ \\
\cline { 2 - 4 } & $\begin{array}{c}\mathrm{x}_{1}=-\frac{1}{a_{1}} \frac{1}{\mathrm{~F}}, \quad \mathrm{x}_{2}=-\frac{a_{1}}{a_{2}} \mathrm{~F} \\
\mathrm{x}_{2}=\frac{\mathrm{Q}^{2}}{\mathrm{~F}^{2}}\end{array}$ & $\begin{array}{c}\mathrm{x}_{1}=-\frac{1}{a_{1}}, \quad \mathrm{x}_{2}=-\frac{a_{1}}{a_{2}} \\
\mathrm{x}_{2}\end{array}$ \\
\hline Factorization: & $\frac{\mathrm{y}}{\mathrm{c}}=\left(1+a_{1} \mathrm{Fx}\right)\left(1+\frac{a_{2}}{a_{1} \mathrm{~F}} \mathrm{x}\right)$ & $\frac{\mathrm{y}}{c}=\left(1+a_{1} \mathrm{x}\right)\left(1+\frac{a_{2}}{a_{1}} \mathrm{x}\right)$ \\
\hline
\end{tabular}

Table 2. Improved quadratic formulas in terms of the two parameters $a_{1}, a_{2}$.

Table 3. Improved quadratic formulas in terms of the single parameter $\mathrm{Q}$ that determines the nature of the roots.

$$
\mathrm{A}=\frac{1}{1+\frac{1}{\mathrm{Q}}\left(\frac{\mathrm{s}}{\omega_{0}}\right)+\left(\frac{\mathrm{s}}{\omega_{0}}\right)^{2}}
$$

where $\omega_{0}$ is the corner frequency (double pole) and $Q$ is determined by various damping resistances. Numerous textbooks and handbooks give graphs of $|A|$ with $Q$ as parameter, but usually only for the underdamped $(Q>0.5)$ case. Figure 4 shows three typical cases: $Q>0.5$ (complex roots); $Q$ $<0.5$ (real roots); and $\mathrm{Q}<0.5$ (well-separated real roots). Features often neglected are: the value of $|A|$ at the corner

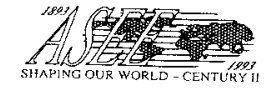

1992 Frontiers in Education Conference 


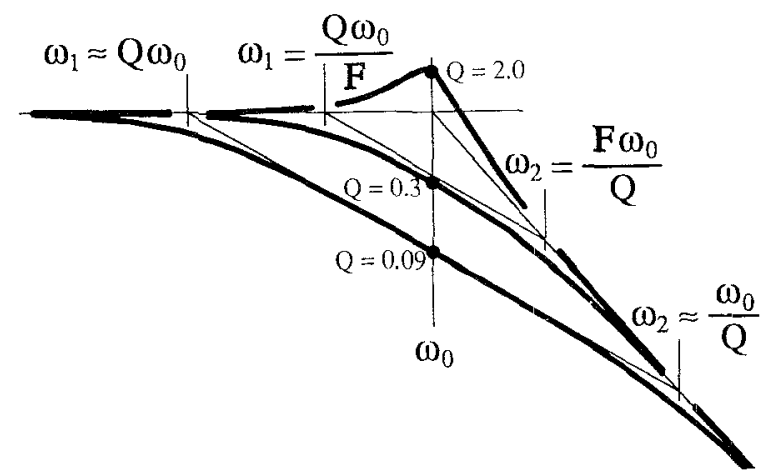

Fig.4. Possible transfer function responses of a lowpass filter having a quadratic pair of poles. The Low Entropy real roots for $\mathrm{Q} \leq 0.5$ become even simpler when $\mathrm{Q}<<0.5$, causing $\mathrm{F} \rightarrow 1$.

frequency is $\mathrm{Q}$, regardless of the value of Q; real poles of $\mathrm{A}$ appear as corner frequencies $\mathrm{Q} \omega_{0} / \mathrm{F}$ and $\mathrm{F} \omega_{0} / \mathrm{Q}$; well-separated real poles of $\mathrm{A}$ appear as corner frequencies $\mathrm{Q} \omega_{0}$ and $\omega_{0} / \mathrm{Q}$. Whether real poles are well-separated or not, they appear equidistant (on a log frequency scale) below and above the corner frequency $\omega_{0}$. Again, the simple Low Entropy expressions for real roots are particularly useful in a design context, in which the roots may be specified and the design problem may be to realize the appropriate values of $\mathrm{Q}$ and $\omega_{0}$.

\section{Conclusions}

It is claimed that the conventional beloved formula for the roots of a quadratic equation suffers from two congenital defects:

Defect \#1: The expressions for the two roots are High Entropy, in that little or no insight is gained into how the quadratic coefficients affect the roots, and substitution of numerical values is the only recourse.

Defect \#2: Real roots are computed with different (and obscure) accuracies, and the accuracy disparity increases the more widely separated are the roots. This results from implicit use of an algorithm that requires computation of the small difference of two almost equal numbers, causing multiplication of calculator roundoff error. In extreme cases, the accuracy of one root can be totally destroyed.

An example is given intended to destroy, instead, students' faith in the conventional formula. The foundation is thereby prepared for their acceptance of an improved formula for quadratic roots that simultaneously overcomes both defects.

The improved formula, exact regardless of whether the roots are real or complex, offers the following benefits.

Real roots can be computed with the same accuracy, and the accuracy is constrained to last-digit roundoff error of the calculator that is employed.

Analytically, both roots can be expressed as simple ratios of the original quadratic coefficients, and a dimensionless factor $F$ that is a unique function of the single parameter $Q$ that determines the nature of the roots (Fig. 1). The radical sign, that dominates the conventional root formula, is confined within the factor $F$ and does not appear elsewhere in the improved root formula. If $\mathrm{Q}>0.5, \mathrm{~F}$ is complex and the roots are a complex pair. If $Q \leq 0.5, F$ is real and the roots are a real pair, equal if $Q$ $=0.5$, and separating (on a $\log$ frequency scale, symmetrically) below and above their equal value for $Q=0.5$ as $Q$ drops below 0.5 .

The ultimate benefit of the improved Low Entropy Expression for quadratic roots is realized in the limiting case when real roots are sufficiently well-separated that $Q<<0.5$, in which case the factor $F$ approaches 1 . As a result the radical sign, confined within $F$, disappears and the roots reduce to simple ratios of the original quadratic coefficients. As a consequence, the quadratic factors directly into a simple product without radical signs. From a design point of view, this is a significant advantage, and a network example is discussed in some detail.

There are many Low Entropy formats for a quadratic equation, the preferred alternative being determined by the structure of the original equation and the structures of the desired roots. Three useful representations are displayed in Tables 1, 2, and 3. Common to all alternatives is the unique function $F$ of $Q$, illustrated in Fig. 1.

There is nothing "new" in anything presented here. The benefits of dealing with normalized variables, leaving a single parameter $Q$, have been recommended, for example, by Waldhauer [2]. It is not to be expected that anything fundamentally new could emerge in relation to the quadratic equation, so basic to all the physical sciences.

The purpose here has been simply to present a different pedagogical perspective, from the viewpoint of Design-Oriented Analysis in terms of Low Entropy Expressions. Analysis is only valuable if it can be "inverted" for design, and from an engineer's standpoint the name of the game is to extract the maximum benefit from the minimum amount of algebra.

Many topics are repeated with higher levels of sophistication during a typical engineering curriculum, as student insight and understanding matures. Somehow, the quadratic equation, probably the first algebraic formula encountered, is considered so simple and basic that no further consideration is warranted.

It's as though a teenager's memory has the famous formula of Eq. (1) burned into it, and subsequently becomes almost a Read Only Memory from which it is very difficult to dislodge this formula, in spite of the fact that much improved versions are well-known to exist, and are worth revisiting.

\section{$\underline{\text { References }}$}

[1] R.D.Middlebrook, "Low-Entropy Expressions: The Key to Design-Oriented Analysis," Proc. IEEE Frontiers in Education, Twenty-First Annual Conference, Purdue University, September 21-24, 1991; 399-403.

[2] Fred D. Waldhauer, Feeaback, John Wiley \& Sons, New York, 1982.

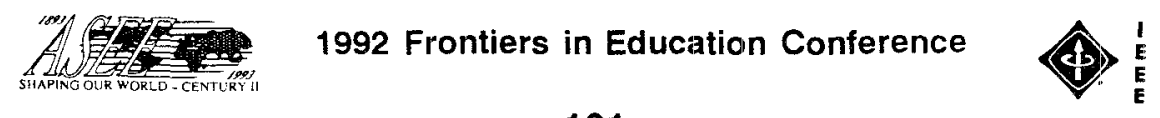




\section{R.D. Middlebrook}

R. D. Middlebrook received the B.A. and M.A. degrees from Cambridge University, England, and the M.S. and Ph.D. degrees from Stanford University, Stanford, CA. $\mathrm{He}$ is a Fellow of the IEEE, and a Fellow of the IEE (U.K.).

$\mathrm{He}$ is Professor of Electrical Engineering at the California Institute of Technology (Caltech). His publications include numerous papers, a book on solid-state device theory, and another on differential amplifiers. He is especially interested in design-oriented circuit analysis and measurement techniques which he teaches at Caltech, and he has conducted short courses on his methods in both Europe and the United States.

Dr. Middlebrook is the recipient of the 1982 IEEE William E. Newe 17 Power Electronics Award for Outstanding Achievement in Power Electronics, and a 1982 Award for Excellence in Teaching, presented by the Board of Directors of the Associated Students of Caltech. In 1984 he received an IEEE Centennial

Medal, and in 1991 he was awarded the Edward Longstreth Medal of the Franklin Institute. 